\title{
Women with Newly Diagnosed Uterine Fibroids: Treatment Patterns and Cost Comparison for Select Treatment Options
}

\author{
Machaon M. Bonafede, PhD, MPH, Scott K. Pohlman, MS,2 Jeffrey D. Miller, MS, \\ Ellen Thiel, MPH, Kathleen A. Troeger, $\mathrm{MPH}^{2}$, and Charles E. Miller, $\mathrm{MD}^{3}$
}

\begin{abstract}
The primary objective of this study was to describe surgical treatment patterns among women with newly diagnosed uterine fibroids (UF). A secondary objective was to estimate the medical costs associated with other common surgical interventions for UF. Claims-based commercial and Medicare data (2011-2016) were used to identify women aged $\geq 30$ years with continuous enrollment for at least 12 months before and after a new diagnosis of UF. Receipt of a surgical or radiologic procedure (hysterectomy, myomectomy, endometrial ablation, uterine artery embolization, and curettage) was the primary outcome. Health care resource utilization and costs were calculated for women with at least 12 months of continuous enrollment following a UF surgical procedure. Among women who met selection criteria, $31.7 \%$ of patients underwent a surgical procedure; $20.9 \%$ of these underwent hysterectomy. An increase was observed over time in the percentage of women undergoing outpatient hysterectomy (from $27.0 \%$ to $40.2 \%$ ) and hysteroscopic myomectomy (from $8.0 \%$ to $11.5 \%$ ). The cost analysis revealed that total health care costs for hysteroscopic myomectomy $(\$ 17,324)$ were significantly lower $(P<0.001)$ than those for women who underwent inpatient hysterectomy $(\$ 24,027)$ and those for women undergoing the 3 comparison procedures. Hysterectomy was the most common surgical intervention. Patients undergoing inpatient hysterectomy had the highest health care costs. Although less expensive, minimally invasive approaches are becoming more common; they are performed infrequently in patients with newly diagnosed UF. The results of this study may be useful in guiding decisions regarding the most appropriate and cost-effective surgical treatment for UF.
\end{abstract}

Keywords: uterine fibroids, surgical intervention, treatment patterns, cost comparison

\section{Introduction}

$\mathbf{U}$ TERINE FIBROIDS (UF) are the most common benign pelvic tumor, affecting more than one half of reproductive-age women in the United States. ${ }^{1,2}$ Although UF are often asymptomatic, $20 \%-50 \%$ of women experience menorrhagia, anemia, recurrent pregnancy loss, the sensation of pelvic pressure, and/or uterine pain. ${ }^{1,3}$ Major risk factors include variation in hormone levels, increasing age up to menopause, and ethnicity. ${ }^{3} \mathrm{Ob}-$ servational data suggest that dietary patterns also may contribute to the likelihood of developing fibroids (eg, consumption of high-fat dairy products, high alcohol consumption). ${ }^{4}$
The numerous treatment options for UF range from pharmacotherapy to radiologic procedures and surgery. ${ }^{5,6}$

\section{Pharmacologic agents}

In general, pharmacologic agents are used to provide relief for patients with mild symptoms, including combined oral contraceptives, progesterone (oral, injection, or intrauterine device), nonsteroidal anti-inflammatory drugs, antifibrinolytics, gonadotropin-releasing hormone agonists, selective estrogen or progesterone receptor modulator progestins, Danazol and aromatase inhibitors. Some of these medications are also useful in reducing tumor growth. ${ }^{7}$ Radiologic and surgical interventions

\footnotetext{
${ }^{1}$ Truven Health Analytics, an IBM Company, Cambridge, Massachusetts.

${ }^{2}$ Hologic, Inc., Marlborough, Massachusetts.

${ }^{3}$ Advocate Lutheran General Hospital, Park Ridge, Illinois.
} 
are typically employed to treat patients with moderate to severe symptoms. ${ }^{7}$

\section{Hysterectomy}

Hysterectomy is the most commonly used surgical procedure for UF; in fact, more than one third of all hysterectomies in the United States are performed to treat benign fibroids. ${ }^{6,8,9}$ Although laparoscopic and robotic assisted hysterectomy for the removal of fibroids are associated with minimal complications and rapid recovery times, these procedures are not feasible options for women who wish to preserve their fertility. ${ }^{10,11}$

\section{Non-hysterectomy clinical management}

Non-hysterectomy clinical management of UF underwent a discernible shift with the introduction of minimally invasive radiologic and surgical procedures. At present, minimally invasive radiologic options include (1) uterine artery embolization (UAE) and (2) magnetic resonance-guided focused ultrasound. Minimally invasive surgical options include (1) laparoscopic myomectomy, (2) robotic assisted myomectomy, (3) hysteroscopic myomectomy, (4) uterine artery occlusion, and (5) laparoscopic radiofrequency ablation. ${ }^{1,3}$ Compared with the more conventional hysterectomy, these treatments offer greater value for patients who wish to preserve fertility; however, symptom relief is not as durable because of risk of recurrence. ${ }^{2,12,13}$

Because there is relatively little real-world evidence available regarding treatment patterns and the costs associated with the medical management of UF, a retrospective trend analysis was conducted in this study to define treatment patterns among women with newly diagnosed UF and to evaluate the relative health care costs associated with radiologic and surgical interventions in real-world settings. A secondary analysis was conducted in this study to compare costs associated with several minimally invasive outpatient treatment options.

\section{Methods}

\section{Study design and data source}

This retrospective, observational analysis utilized claimsbased data from a commercially insured population (Truven Health MarketScan Commercial Claims and Encounters) and a population of Medicare beneficiaries (Medicare Supplemental and Coordination of Benefits) for the calendar years 2011 through 2016. These 2 databases contain complete longitudinal records of inpatient and outpatient services, and prescription drug claims for millions of individuals with commercial insurance or Medicare supplemental insurance paid by employers. Both data sources include health care costs, utilization, and outcomes data for health care services performed in inpatient and outpatient settings. Medical claims are linked to outpatient prescription drug claims and person-level enrollment data via unique enrollee identifiers. All data conform to Health Insurance Portability and Accountability Act of 1996 confidentiality requirements; therefore, institutional review board approval was unnecessary.

\section{Patient selection}

Women aged 30 years or older with a new diagnosis of UF (International Classification of Diseases, Ninth Revision, Clinical Modification]: 218.x) between January 1, 2011, to December 31, 2015, were eligible for inclusion in the general trend analysis. The date of the first UF diagnosis was set as the index date. Continuous enrollment, with both medical and pharmacy benefits, was required for the 12 months before (pre-index period) and after the index date (post-index period). Exclusions included patients with UF diagnoses or a surgical procedure in the 12 months pre-index period or gynecological cancer during the study period. Hysterectomy patients with evidence of an enlarged uterus $(>250 \mathrm{~g})$ were excluded based on procedure codes indicating uterine weight $>250 \mathrm{~g}$; uterine weight is not included in procedure coding for other UF interventions.

A subset of patients was identified for a secondary analysis to evaluate health care costs and utilization associated with select interventions. The sample included women who underwent hysterectomy, hysteroscopic myomectomy, or UAE from January 1, 2012, to December 31, 2015, and had at least 12 months pre- and post-index continuous enrollment in the Truven Health MarketScan Commercial Claims and Encounters database. The date of the surgical intervention served as the index date.

\section{Outcome measures}

The use of diagnostic and treatment procedures and pharmacotherapy, as identified by administrative claims, were evaluated in the 12-month period following the initial UF diagnosis. The general trend analysis evaluated the full range of gynecologic surgical interventions common among women with UF, even those commonly performed for reasons beyond the treatment of fibroids: all types of hysterectomy and myomectomy, UAE, endometrial ablation, and curettage (cervix or uterus). The proportion of patients treated with hormone therapy and intrauterine devices also was recorded. For the secondary cost analysis, health care utilization and costs during the first 30 days and the 12month follow-up period were recorded for patients who underwent one of the following procedures: inpatient hysterectomy, outpatient hysterectomy, outpatient hysteroscopic myomectomy, or outpatient UAE. Both all-cause and obstetrician/gynecologist (OB/GYN)-related costs, which include the costs of reinterventions or complications during the follow-up period, are described in the analysis results reported here. The outpatient hysterectomy cohort was further segmented to analyze patients who underwent outpatient vaginal hysterectomies without additional surgical repair as represented by 2 Current Procedural Terminology codes: 58260 and 58262. The age of patients who underwent any of the selected surgical interventions was recorded on the index date as a study covariate.

\section{Statistical analysis}

Descriptive analysis was employed for all study variables. Categorical variables were summarized by frequency and percentages, and continuous variables were reported as means and standard deviations (SD). $t$ Tests and Fisher exact 
tests were used to evaluate the statistical significance and a $P$ value of $<0.05$ was considered statistically significant.

\section{Results}

\section{Sample size}

Of 50,216,361 women in the MarketScan database, 310,769 women with newly diagnosed UF met the selection criteria (Fig. 1). Stratification of patients by year of diagnosis yielded 5 mutually exclusive cohorts: 76,751 in 2011; 68,878 in $2012 ; 61,694$ in $2013 ; 53,477$ in 2014 , and 49,969 in 2015.

For the secondary utilization and cost analysis, 107,708 women met all the eligibility criteria. The outpatient hysterectomy cohort consisted of 44,682 patients (5030 of these patients received vaginal hysterectomy without additional surgical repair); the inpatient hysterectomy cohort consisted of 45,119 patients; the hysteroscopic myomectomy cohort consisted of 13,323 patients; and the UAE cohort consisted of 4584 patients. Patients who underwent UAE were slightly younger $(44.5$ years $[\mathrm{SD}=5.3])$ than those who underwent outpatient hysterectomy $(46.8$ years $[\mathrm{SD}=7.8])$, inpatient hysterectomy $(47.3$ years $[\mathrm{SD}=7.7])$, or hysteroscopic myomectomy $(46.3$ years $[\mathrm{SD}=8.8])$.

\section{General trend analysis: pattern of surgical or radiological treatment}

Overall, $31.7 \%(\mathrm{~N}=98,554)$ of patients underwent a surgical or radiological procedure for UF within 1 year of a new diagnosis. The proportion of patients receiving any surgical or radiological procedure decreased significantly from $34.8 \%$ of patients diagnosed in 2011 to $26.0 \%$ in 2015 $(P<0.001)$. Hysterectomy was the most commonly performed procedure $(20.9 \%)$ within 1 year of UF diagnosis, with annual rates decreasing from $22.9 \%$ of patients diagnosed in 2011 to $17.0 \%$ in $2015(P<0.001)$. Other surgical interventions included: any myomectomy $(5.1 \%)$, endometrial ablation $(4.3 \%)$, and curettage procedures $(3.2 \%)$. The yearly rates of myomectomy among all women diagnosed

Patients with at least one non-diagnostic medical claim with a diagnosis of uterine fibroids (ICD9-CM 218.x) during year indicated. Earliest medical claim was set as the index date $\mathrm{n}=1,115,805(2.2 \%)$

Patients who were $\geq 30$ years of age on the index date $n=1,081,735(96.9 \%)$

Patients with continuous enrollment for at least 12 months before the index date $n=757,442(67.9 \%)$
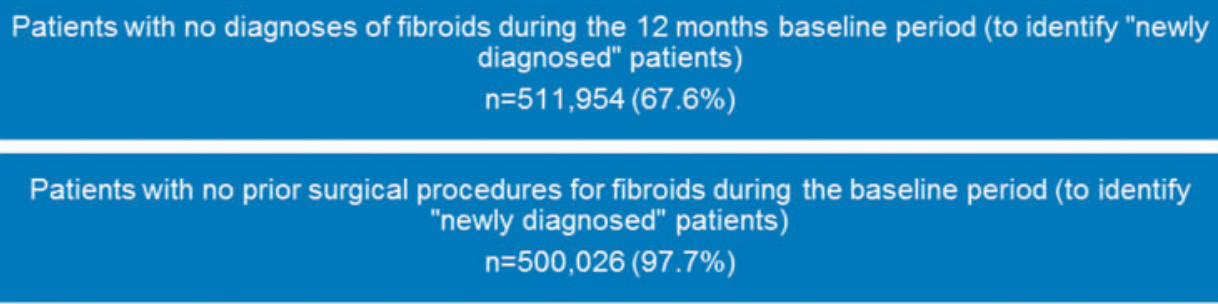

Patients with continuous enrollment for at least 12 months after the index date $n=367,576(73.5 \%)$

Patients with no diagnoses of gynecological cancers during the study period $\mathrm{n}=358,106(97.4 \%)$

Patients with no evidence of large $(>250 \mathrm{~g})$ uterus in study period $\mathrm{n}=339,717(94.9 \%)$

FIG. 1. Patient selection. ICD-9-CM, International Classification of Diseases, Ninth Revision, Clinical Modification. 
with UF were relatively stable $(4.8 \%$ to $5.4 \%)$. The proportion of patients who underwent curettage procedures mirrored the overall surgical trend, with prevalence decreasing from $3.6 \%$ in 2011 to $2.8 \%$ in 2015 . UAE was relatively uncommon $(<1.0 \%$ each year) (Fig. 2$)$.

Of all patients with surgical or radiological interventions, an average of $65.8 \%$ patients underwent hysterectomy following a UF diagnosis from 2011 through 2015. The rates for specific minimally invasive procedures were considerably lower: $14.3 \%$ underwent endometrial ablation; $10.4 \%$ underwent curettage; $8.9 \%$ underwent hysteroscopic myomectomy; $3.9 \%$ underwent abdominal myomectomy; $3.4 \%$ underwent laparoscopic/robotic myomectomy; and $2.1 \%$ of patients underwent UAE. Of the 3 myomectomy procedure options (ie, abdominal myomectomy, laparoscopic/robotic assisted myomectomy, hysteroscopic myomectomy), hysteroscopic myomectomy accounted for an increasing proportion of patients with surgical interventions over time (from $8.0 \%$ in 2011 to $11.5 \%$ in 2015) (Fig. 3).

Overall, $11.8 \%$ of patients used hormone therapy after being diagnosed during the 2011-2015 period, with a slight increase in utilization from $11.2 \%$ in 2011 to $13.2 \%$ in 2015.

\section{Secondary analysis: health care costs and utilization}

The secondary analysis consisted of 45,119 women who underwent inpatient hysterectomy and 62,589 women who were treated with one of the selected outpatient procedures (hysterectomy, hysteroscopic myomectomy, UAE). Of the women treated with outpatient procedures, $71.3 \%$ underwent outpatient hysterectomy, $21.2 \%$ underwent hysteroscopic myomectomy, and $7.3 \%$ underwent UAE. Of the 44,682 patients who underwent outpatient hysterectomy, only 5030 $(11.2 \%)$ of these procedures were performed vaginally (without additional surgical repair).

Of the 4 cohorts in the secondary analysis, the patients treated with hysteroscopic myomectomy incurred the lowest total health care costs in the 0 to 29 days following surgery (\$8261 vs. $\$ 16,549$ for inpatient hysterectomy, vs. $\$ 14,416$ for outpatient hysterectomy, vs. $\$ 11,982$ for outpatient vaginal hysterectomy, and vs. $\$ 15,140$ for UAE). In the 30 to 365 days following surgery, the hysteroscopic myomectomy cohort incurred slightly higher total payments (\$9062 vs $\$ 7478$ for inpatient hysterectomy, \$7394 outpatient hysterectomy, $\$ 7157$ for outpatient vaginal hysterectomy, and $\$ 7199$ for UAE) (Fig. 4). However, the mean total annual payments were lowest for the hysteroscopic myomectomy cohort $(\$ 17,324)$ compared to those who underwent inpatient hysterectomy $(\$ 24,027)$, outpatient hysterectomy $(\$ 21,810)$, outpatient vaginal hysterectomy $(\$ 19,138)$, and UAE $(\$ 22,339)$.

Within the first month after surgery, OB/GYN-related costs for the hysteroscopic myomectomy cohort (\$6288) were approximately one half that of the inpatient hysterectomy cohort $(\$ 15,099)$, the outpatient hysterectomy cohort $(\$ 12,403)$, the outpatient vaginal hysterectomy cohort $(\$ 10,550)$, and the UAE cohort $(\$ 11,764)$ (Fig. 5). Although the hysteroscopic myomectomy cohort posted the highest OB/GYN-related costs during the 30-365 days following surgery, total OB/GYN-related costs (\$9584) were lower than those for the inpatient hysterectomy $(\$ 16,414)$, outpatient hysterectomy $(\$ 13,588)$, outpatient vaginal hysterectomy $(\$ 11,885)$, and UAE $(\$ 14,005)$ cohorts.

Of the interventions evaluated in the cost analysis, the hysterectomy cohorts experienced higher out-of-pocket payments within the first 7 days of surgery ( $\$ 1442$ for inpatient hysterectomy, $\$ 1457$ for outpatient hysterectomy, and \$1416 for outpatient vaginal hysterectomy) when compared with the hysteroscopic myomectomy (\$1056) and UAE (\$1123) cohorts.

Overall, 5.6\% of women in the outpatient hysterectomy cohort and $3.9 \%$ of women in the outpatient vaginal hysterectomy cohort required an inpatient stay in the 0 to 29 days following surgery. Of the women with an inpatient stay, $68.4 \%$ in the outpatient hysterectomy and $74.9 \%$ in the outpatient vaginal hysterectomy cohort had an OB/GYNrelated diagnosis. The percentages of patients requiring inpatient stays in the other 2 cohorts were: $2.5 \%$ for hysteroscopic myomectomy and $10.4 \%$ for UAE. Of these,

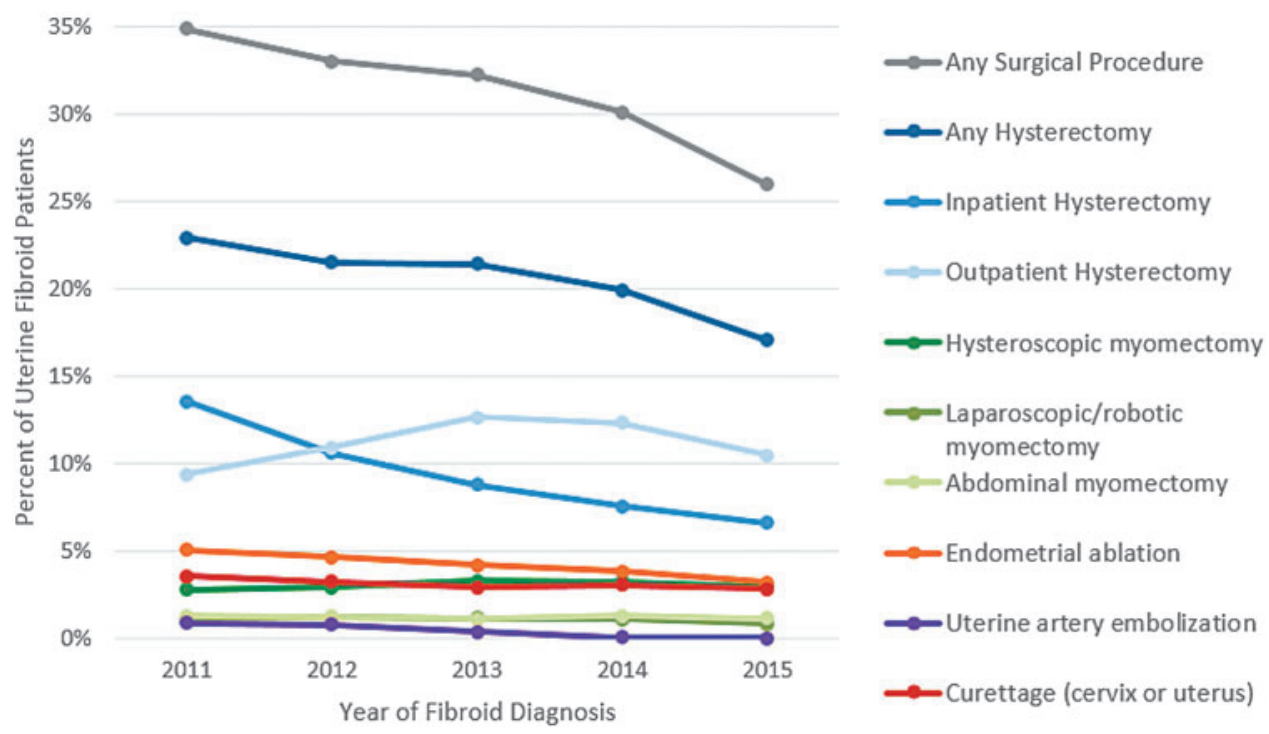

FIG. 2. Pattern of surgical treatments in women diagnosed from 2011-2015. 


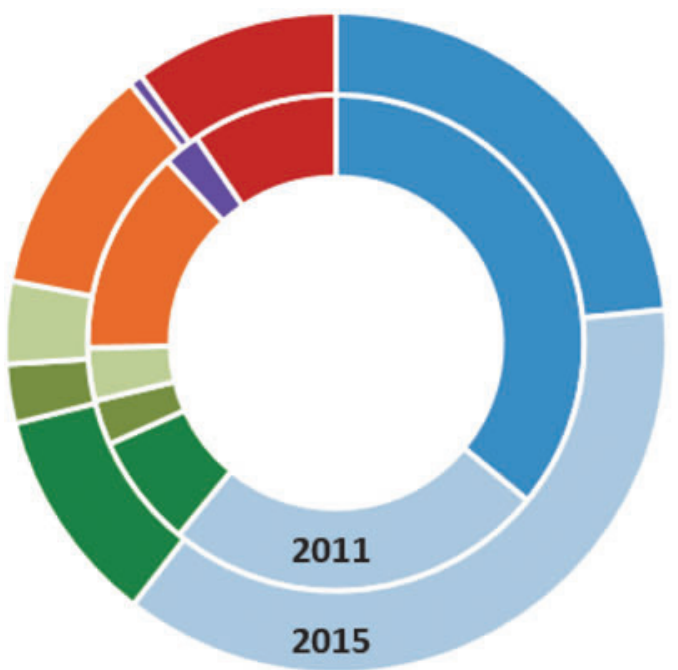

= Inpatient Hysterectomy

= Outpatient Hysterectomy

- Hysteroscopic myomectomy

- Laparoscopic/robotic myomectomy

= Abdominal myomectomy

- Endometrial ablation

- Uterine artery embolization

- Curettage (cervix or uterus)

FIG. 3. Prevalence of procedures among women who received surgical treatment (2011 and 2015).

$33.3 \%$ in the hysteroscopic myomectomy cohort and $53.3 \%$ of UAE cohort had an OB/GYN-related diagnosis. The results of the cost analysis are summarized in Table 1.

\section{Discussion}

Given the prevalence of UF and the wide variety of possible surgical treatment options, it is important to understand the costs and benefits associated with each as they pertain to patients in a real-world setting. Although there are multiple reasons for women to undergo a particular type of treatment, including the nature of the pathology, patient preference, and surgeon experience, there is evidence supporting the use of minimally invasive treatments to remove UF to lower complication rates, improve quality of outcomes, and reduce costs compared with hysterectomy procedures. ${ }^{14-17}$
Several US studies have evaluated the economic burden associated with UF treatment. ${ }^{18-23}$ One systematic review estimated the annual economic burden of UF in the United States to be between $\$ 5.9$ and $\$ 34.4$ billion per year (in 2010 US dollars), including direct and indirect costs and costs associated with obstetric complications. ${ }^{22}$ Another systematic review of 26 studies (19 from the United States) conducted between 2000 and 2013 estimated total direct and indirect costs to be $\$ 11,717$ to $\$ 25,023$ per patient per year, following diagnosis of or surgery for UF patients. The additional total cost per patient in the first year post diagnosis ranged from $\$ 2200-\$ 15,952 .^{23}$

The present analysis demonstrates that surgery is frequently performed within 12 months of a UF diagnosis, with hysterectomy being the most common surgical procedure. However, the trend analysis suggests that surgery is

\section{Total Payments}

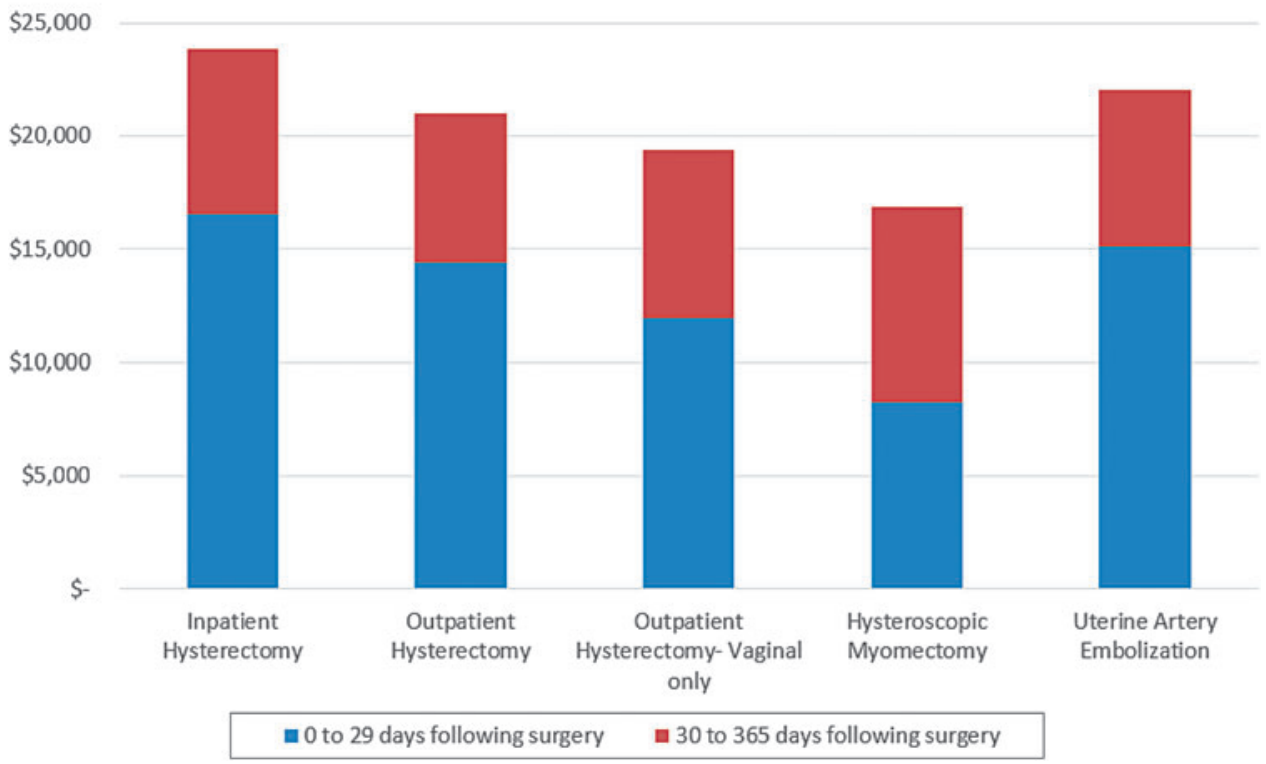

FIG. 4. Total health care costs associated with surgical interventions in the 1-year follow-up period. 


\section{OB/GYN-Related Payments}

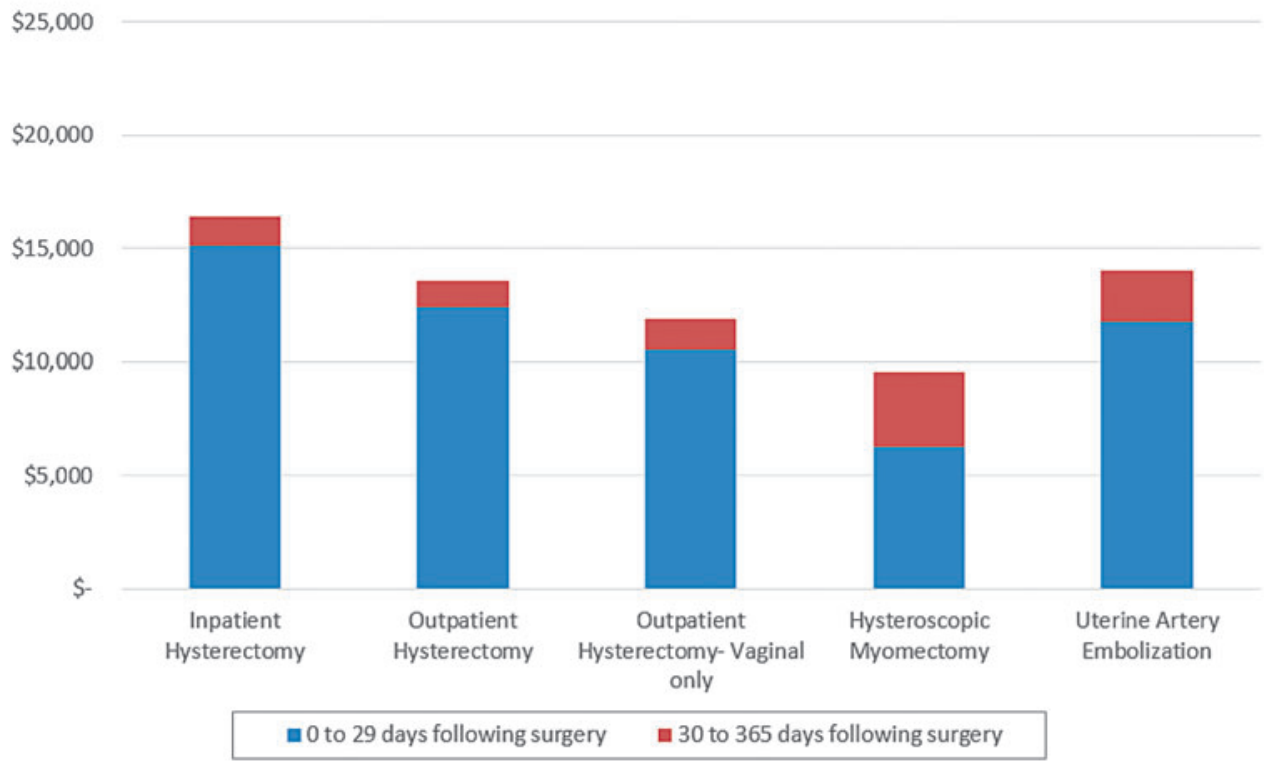

FIG. 5. Obstetrician/gynecologist-related health care costs associated with surgical interventions in the 1-year follow-up period.

becoming slightly less common among women with newly diagnosed fibroids; $34.8 \%$ of women diagnosed in 2011 were treated surgically while only $26.0 \%$ of women diagnosed in 2015 were treated surgically. This analysis also demonstrates a recent trend toward less invasive procedures for women who undergo surgery. In particular, there has been an increase in the percentage of hysterectomies performed as outpatient procedures. For women diagnosed with UF in $2011,41.0 \%$ of hysterectomy procedures were performed in outpatient settings. This increased to $61.3 \%$ for women diagnosed in 2015. Additionally, although rates of hysteroscopic myomectomy remain relatively low in women with newly diagnosed fibroids $3.0 \%$ over the entire study period), this minimally invasive procedure saw a $44 \%$ increase between 2011 and 2015 among the group of women who were treated surgically.

The secondary analysis indicates that hysterectomy is less costly when performed as an outpatient procedure than as an inpatient procedure. Furthermore, both vaginal hysterectomy and hysteroscopic myomectomy were less costly than outpatient hysterectomy overall (including vaginal hysterectomy). Outpatient vaginal hysterectomy and hysteroscopic myomectomy also were associated with lower rates of subsequent inpatient stays than outpatient hysterectomy (overall) and UAE. Given the current, relatively limited use of vaginal hysterectomy and hysteroscopic myomectomy, there may be an opportunity to reduce overall health care costs by increasing use of these minimally invasive procedures for appropriate patients. Although some procedures, such as hysterectomy and UAE, offer a broader treatment population (eg, management of intramuscular and subserosal fibroids) than hysteroscopic myomectomy, it is likely that some women may be candidates for more than 1 type of surgical treatment. As insurance claims data alone are insufficient to make determinations of which individual patients may be candidates for specific alternative treatments, these data cannot be used to accurately estimate the percentage of women who could potentially be treated with less costly interventions.

\section{Limitations}

Limiting the analysis to a 12-month period following fibroid diagnosis may affect the types of procedures that are reported. For example, UAE may be underrepresented in the initial 12 months following diagnosis, as the time from diagnosis to treatment may be longer for UAE than for some other procedures. Future research should expand the time period of the cost analysis to identify longer term costs associated with index treatments, potential complications, reinterventions, and other sequelae.

This study has the usual limitations inherent with administrative claims data. Misclassification of UF, covariates, or study outcomes may have occurred because patients were identified through administrative claims data rather than medical records. All claims databases rely on administrative claims data that are generated for the purposes of facilitating payment. Claims data lack potentially important drivers of disease and treatment costs, including physician skill and myoma characteristics, as well as a patient's potential desire to preserve fertility. Likewise, the treatments compared in this analysis may not be available to all patients because of availability or physician training, neither or which could be controlled for in this current analysis. Further, the exclusion of women with uterine weight greater than $250 \mathrm{~g}$ was only applicable to women with hysterectomy as available codes do not include uterine weight for the other procedures. This analysis was conducted among women with commercial health insurance coverage or private Medicare supplemental coverage and thus may not be generalizable to patients with Medicaid or those without insurance. Finally, the results of 





the study may be prone to bias from unobservable or confounding factors affecting choice of surgery and the outcomes of interest.

\section{Conclusions}

Hysterectomy was the most common surgical intervention within 1 year of UF diagnosis and inpatient hysterectomy had the greatest health care costs following surgery. Less expensive, minimally invasive approaches such as outpatient vaginal hysterectomy and hysteroscopic myomectomy are becoming more common, but still are performed infrequently in patients with newly diagnosed fibroids. These results can help inform future medical decision making around cost-effective decisions for the treatment of uterine fibroids.

\section{Author Disclosure Statement}

Dr. Bonafede, Mr. Miller, and Mrs. Thiel are employees of Truven Health Analytics, an IBM Company, which received funding from Hologic, Inc. to conduct this study. Mr. Pohlman and Ms. Troeger are employees of Hologic, Inc., which funded this study. Dr. Miller has a stock option agreement with Halt Medical, Inc., is a consultant for Medtronic, and has received research funding from Allergen, Espiner Medical, Gynesonics, Karl Storz GmbH, and Myovant Sciences, Inc. This study was sponsored by Hologic Inc., Marlborough, MA.

\section{References}

1. Silberzweig JE, Powell DK, Matsumoto AH, Spies JB. Management of uterine fibroids: a focus on uterine-sparing interventional techniques. Radiology 2016;280:675-692.

2. Chen S, Pitre E, Kaunelis D, Singh S. Uterine-Preserving Interventions for the Management of Symptomatic Uterine Fibroids: A Systematic Review of Clinical and CostEffectiveness. https://www.ncbi.nlm.nih.gov/pubmedhealth /PMH0086153/pdf/PubMedHealth_PMH0086153.pdf Accessed February 15, 2017.

3. Laughlin-Tommaso SK. Alternatives to hysterectomy: management of uterine fibroids. Obstet Gynecol Clin North Am 2016;43:397-413.

4. Stewart EA. Uterine fibroids. N Engl J Med. 2015;372: 1646-1655.

5. Sabry M, Al-Hendy A. Medical treatment of uterine leiomyoma. Reprod Sci 2012;19:339-353.

6. Singh SS, Belland L. Contemporary management of uterine fibroids: focus on emerging medical treatments. Curr Med Res Opin 2015;31:1-12.

7. Barrett ML, Weiss AJ, Stocks C, Steiner CA, Myers ER. Procedures to Treat Benign Uterine Fibroids in Hospital Inpatient and Hospital-Based Ambulatory Surgery Settings, 2013. HCUP Statistical Brief \#200. Rockville, MD: Agency for Healthcare Research and Quality; January 2016. www. hcup-us.ahrq.gov/reports/statbriefs/sb200-Procedures-TreatUterine-Fibroids.pdf Accessed February 15, 2017.

8. Khan AT, Shehmar M, Gupta JK. Uterine fibroids: current perspectives. Int J Womens Health 2014;6:95-114.

9. Bonafede MM, Cappell KA, Pohlman SK, Troeger KA. Treatment patterns for women with newly diagnosed uterine fibroids. JMIG 2014;21(6 suppl):S135.
10. Guo XC, Segars JH. The impact and management of fibroids for fertility: an evidence-based approach. Obstet Gynecol Clin North Am 2012;39:521-533.

11. Fuldeore M, Yang H, Soliman AM, Winkel C. Healthcare utilization and costs among women diagnosed with uterine fibroids: a longitudinal evaluation for 5 years pre- and postdiagnosis. Curr Med Res Opin 2015;31:1719-1731.

12. Mauskopf J, Flynn M, Thieda P, Spalding J, Duchane J. The economic impact of uterine fibroids in the United States: a summary of published estimates. J Womens Health (Larchmt) 2005;14:692-703.

13. Dembek CJ, Pelletier EM, Isaacson KB, Spies JB. Payer costs in patients undergoing uterine artery embolization, hysterectomy, or myomectomy for treatment of uterine fibroids. J Vasc Interv Radiol 2007;18:1207-1213.

14. Hirst A, Dutton S, Wu O, et al. A multi-centre retrospective cohort study comparing the efficacy, safety and costeffectiveness of hysterectomy and uterine artery embolization for the treatment of symptomatic uterine fibroids. The HOPEFUL study. Health Technol Assess 2008;12:1-248, iii.

15. Smeets AJ, Nijenhuis RJ, Boekkooi PF, et al. Safety and effectiveness of uterine artery embolization in patients with pedunculated fibroids. J Vasc Interv Radiol 2009;20:1172-1175.

16. Scheurig-Muenkler C, Lembcke A, Froeling V, Maurer M, Hamm B, Kroencke TJ. Uterine artery embolization for symptomatic fibroids: long-term changes in disease-specific symptoms and quality of life. Hum Reprod 2011;26:20362042.

17. Fennessy FM, Kong CY, Tempany CM, Swan JS. Qualityof-life assessment of fibroid treatment options and outcomes. Radiology 2011;259:785-792.

18. Beinfeld MT, Bosch JL, Gazelle GS. Hospital costs of uterine artery embolization and hysterectomy for uterine fibroid tumors. Acad Radiol 2002;9:1300-1304.

19. Flynn M, Jamison M, Datta S, Myers E. Health care resource use for uterine fibroid tumors in the United States. Am J Obstet Gynecol 2006;195:955-964.

20. Lee DW, Ozminkowski RJ, Carls GS, Wang S, Gibson TB, Stewart EA. The direct and indirect cost burden of clinically significant and symptomatic uterine fibroids. J Occup Environ Med 2007;49:493-506.

21. Goldberg J, Bussard A, McNeil J, Diamond J. Cost and reimbursement for three fibroid treatments: abdominal hysterectomy, abdominal myomectomy, and uterine fibroid embolization. Cardiovasc Intervent Radiol 2007;30:54-58.

22. Cardozo ER, Clark AD, Banks NK, Henne MB, Stegmann BJ, Segars JH. The estimated annual cost of uterine leiomyomata in the United States. Am J Obstet Gynecol 2012; 206:211.e1-211.e9.

23. Soliman AM, Yang H, Du EX, Kelkar SS, Winkel C. The direct and indirect costs of uterine fibroid tumors: a systematic review of the literature between 2000 and 2013. Am J Obstet Gynecol 2015;213:141-160.

Address correspondence to: Machaon M. Bonafede, PhD, MPH Truven Health Analytics, an IBM Company 75 Binney Street Cambridge, MA 02142

E-mail: mbonafed@us.ibm.com 